\title{
Closed-Eye Visual Hallucinations Preceding Severe Alcohol Withdrawal
}

\author{
Saral Desai ${ }^{1}$, Anca E. Toma ${ }^{1}$, Artem Sunik ${ }^{2}$ \\ 1. Department of Psychiatry, Brookdale University Hospital Medical Center, Brooklyn, USA 2. Department of \\ Neurology, Brookdale University Hospital Medical Center, Brooklyn, USA
}

Corresponding author: Saral Desai, ssaralix@gmail.com

\begin{abstract}
Few existing cases of closed-eye visual hallucinations have been reported. These rare perceptual disturbances are distinct from open-eye visual hallucinations, as observed in Charles Bonnet syndrome. This case report discusses a 35-year-old male who presented with closed-eye visual hallucinations 24 hours before severe alcohol withdrawal. On initial presentation, the patient denied auditory or visual hallucinations. The day before the onset of severe alcohol withdrawal, the patient reported vivid, colorful, and lifelike visual hallucinations with his eyes closed which disappeared with eyes open. The hallucinations consisted of cartoon characters such as Daffy Duck, a lifelike blond woman with curly hair, and a treecovered landscape. The patient endorsed awareness that the hallucinations were not real. The next day, the patient became acutely delirious with rapid onset of agitation, space-time disorientation, open-eye visual hallucinations, and emotionally disturbing illusions. The patient was promptly transferred to the intensive care unit (ICU) to manage severe alcohol withdrawal, including administration of intravenous sedation. During follow-up evaluation, the patient retained a clear memory of the closed-eye hallucinations. However, he had no recollection of the ICU course. Several etiologies were considered, including alcohol withdrawal complicated by polysubstance withdrawal and acute hepatitis, which resolved following pharmacological treatment. To the best of our knowledge, this is the first case report of its kind. Additional research is required to elucidate the etiology, mechanism, and clinical implications of closed-eye visual hallucinations.
\end{abstract}

Review began 07/14/2021 Review ended 08/03/2021 Published 08/09/2021

\section{() Copyright 2021}

Desai et al. This is an open access article distributed under the terms of the Creative Commons Attribution License CC-BY 4.0., which permits unrestricted use, distribution, and reproduction in any medium, provided the original author and source are credited.
Categories: Neurology, Psychiatry

Keywords: visual hallucinations, closed-eye visual hallucinations, alcohol withdrawal, psychiatry, neurology, hallucinations, substance use, delirium tremens, charles bonnet syndrome

\section{Introduction}

Visual hallucinations are common in neurological and psychiatric disorders, such as schizophrenia, bipolar disorder with psychotic features, schizoaffective disorder, delirium, dementia, temporal lobe epilepsy, migraines, sleep disorders, substance use disorders, and neoplastic disorders [1]. Charles Bonnet syndrome is a known condition in which visual hallucinations occur due to visual sensory deprivation caused by vision loss and is most prevalent in elderly patients [2]. Previously published case reports describe a novel type of visual hallucination occurring primarily with eye closure and are distinct from the visual hallucinations associated with Charles Bonnet syndrome [3-8]. We present a case of closed-eye visual hallucinations with onset before severe alcohol withdrawal. Clinical significance, etiology, and treatment of such closed-eye visual hallucinations remain unknown.

\section{Case Presentation}

A 35-year-old male with prior history of major depressive disorder, traumatic brain injury, and severe polysubstance use (alcohol, cocaine, cannabis, opiates, and benzodiazepines) presented to the emergency department via emergency medical services after attempting to cut his wrists bilaterally in the setting of polysubstance use. In the emergency department, labs were remarkable for a blood alcohol level of 421 $\mathrm{mg} / \mathrm{dL}$ and mild transaminitis (aspartate aminotransferase $325 \mathrm{U} / \mathrm{L}$, alanine transaminase $284 \mathrm{U} / \mathrm{L}$ ). The urine toxicology screen was positive for cannabinoids and benzodiazepines. On initial psychiatric evaluation, the patient was cooperative, tearful, and withdrawn. He endorsed suicidal ideation with depressed mood, anhedonia, hopelessness, and difficulty sleeping. During this time, the patient was alert and oriented to time, place, and person. He denied hallucinations, delusions, or other perceptual disturbances, and no psychosis was observed. The patient was medically cleared and transferred to the inpatient psychiatric unit for further stabilization. Within 72 hours, the patient was found to have worsening transaminitis (>1,500 U/L) and subsequently transferred to the regular medical floor to manage acute hepatitis. At this time, the ammonia level was normal $(9 \mu \mathrm{mol} / \mathrm{L})$, and mentation was intact.

The following day, the patient remained alert and oriented. He was cooperative, friendly, and maintained fair eye contact. His mood was neutral, his thought process logical and linear, and he denied suicidal or homicidal ideation. Physical examination was remarkable for mild bilateral hand tremors. The patient described new-onset closed-eye visual hallucinations, which resolved upon opening his eyes and recurred on eye closure. He denied auditory hallucinations, delusions, or other perceptual disturbance, and none 
were elicited. On further interrogation, the patient stated the hallucinations were vivid, graphic, and animated. He specifically reported observing the cartoon character Daffy Duck when he closed his eyes, which subsequently disappeared when he opened his eyes.

The next morning, the patient became disoriented, agitated, and presented with new-onset illusions, mistaking the treating psychiatrist for his father and the hospital bed for his living room couch. The patient was in severe emotional distress, asking the treatment team to help him save his father, whom he believed was trapped under the couch. The patient became increasingly agitated, attempted to elope by hitting his hospital room window with an IV pole, and was imminently transferred to the intensive care unit (ICU) to treat severe delirium, primarily attributed to alcohol withdrawal in the setting of suspected polysubstance withdrawal. At this time, the ammonia level was found to be elevated at $82 \mu \mathrm{mol} / \mathrm{L}$. The patient was intubated for airway protection and started on intravenous (IV) midazolam $20 \mathrm{mg} /$ hour and fentanyl 2,000 $\mu \mathrm{g} /$ hour.

After 72 hours, the patient was extubated, regained alertness and orientation, and followed complex commands. At this time, the patient denied hallucinations, delusions, illusions, or other perceptual disturbances. To further explore the nature of this unique clinical presentation, we gauged the patient's level of insight regarding the hallucinations during a follow-up evaluation. The patient precisely recalled vivid closed-eye visual hallucinations. He confirmed the absence of visual hallucinations on admission. He also confirmed that the hallucinations began the day before severe delirium, though he stated he had little to no recollection of his ICU course. The patient also confirmed that the hallucinations appeared when he closed his eyes and resolved when he opened his eyes. Each time he closed his eyes, the patient recalled watching a different scene or subject, occurring in nonsequential order, without distinct features. The patient described seeing cartoon characters such as Daffy Duck, an unfamiliar blond woman with curly hair having an ambiguous face, and a forested landscape. He described the images as vibrant, lifelike, and evocative. He denied the images were memories or personally relevant and stated he knew these visual hallucinations were not real. Although the patient admitted to a prior history of delirium tremens, alcohol-related blackouts, polysubstance use, and polysubstance withdrawal, the patient stated the closed-eye visual hallucinations occurred for the first time.

\section{Discussion}

Visual hallucinations are frequently observed in various neurological conditions, including Lewy body dementia, temporal lobe epilepsy, and Parkinson's disease [1]. Additionally, they are observed in psychiatric conditions such as psychosis and substance intoxication or substance withdrawal [1]. Delirium is another condition frequently associated with visual hallucinations [1]. Additionally, hypnagogic and hypnopompic visual hallucinations commonly occur during transitions of wakefulness to sleep and vice versa, respectively [9]. Finally, visual hallucinations may occur in narcolepsy during the transition from rapid eye movement into wakefulness [9].

Closed-eye visual hallucinations are similar to those seen in Charles Bonnet syndrome in that both occur in the absence of visual sensory input [2]. The visual hallucinations described by our patient were silent, noninteractive, and impersonal, much like the ones described in Charles Bonnet syndrome, and differing from the hallucinations characteristic of psychosis [10].

The visual hallucinations described by our patient also differ from those seen in Charles Bonnet syndrome in several ways. First, our patient's vision is intact. Second, he only experienced visual hallucinations upon eye closure. This contrasts with Charles Bonnet syndrome, characterized by visual hallucinations with open eyes that resolve upon eye closure [10]. We consider the possibility that our patient's intact afferent visual input may be dominant when his eyes are open, which may override an internally generated signal in the visual cortex responsible for the visual hallucinations. Charles Bonnet syndrome is characterized by visual impairment resulting in sensory deafferentation, leading to disinhibition of the visual cortex leading to visual hallucinations [11]. Our patient's closed-eye visual hallucinations may have occurred from disinhibition of the visual cortex in the absence of visual input on the eye closure due to an imbalance between excitatory and inhibitory neurotransmitters due to alcohol and polysubstance withdrawal. However, further research is required to determine the exact mechanism and its clinical implications.

At times mistaken for visual hallucinations, phosphenes are spontaneous and luminous intrinsic visual phenomena present as compound geometric shapes [12,13]. In contrast to the content of visual hallucinations, luminous phenomena such as phosphenes or white noise are ambiguous shapes unrelated to cultural references $[12,13]$. This distinction is strengthened when the visual hallucinations are associated with psychoactive substance use, mood disturbance, or in the setting of acute conditions such as fever or psychosis $[12,13]$. Although it cannot be ruled out, we believe our patient's sensory experience is unlikely attributed to phosphenes.

A literature search revealed a limited number of published case reports of closed-eye visual hallucinations at the time of submission, summarized in Table 1. The majority of cases describe patients with closed-eye visual hallucinations in the absence of delirium. Most patients acknowledge that the hallucinations were not real. Most patients describe the hallucinations as vivid, lifelike images, resolving with either correction of 
underlying etiology, or more commonly, spontaneously without treatment. Reported symptom duration ranges between four hours to eleven days.

\begin{tabular}{|c|c|c|c|c|c|}
\hline Year & Author & Type of hallucination & Duration & Suspected etiology & Treatment \\
\hline 2018 & $\begin{array}{l}\text { Peck et al. } \\
\text { [3] }\end{array}$ & $\begin{array}{l}\text { A lifelike colorful moving car driving in front of the } \\
\text { patient }\end{array}$ & Six days & Hyponatremia & $\begin{array}{l}\text { Resolved after correction } \\
\text { of hyponatremia }\end{array}$ \\
\hline 2011 & $\begin{array}{l}\text { Weinschenk } \\
\text { et al. [4] }\end{array}$ & $\begin{array}{l}\text { Vivid and realistic images of glaciers, waterfalls, } \\
\text { and other picturesque landscapes }\end{array}$ & days & Lidocaine use & Spontaneous resolution \\
\hline 2008 & [5] & $\begin{array}{l}\text { VIVId, realistic Images of colored clothes, lace } \\
\text { curtains, handbags, and sofas }\end{array}$ & $\begin{array}{l}\text { Four } \\
\text { days }\end{array}$ & $\begin{array}{l}\text { Postoperative after epıdural } \\
\text { with general anesthesia }\end{array}$ & Spontaneous resolution \\
\hline 2005 & $\begin{array}{l}\text { Eissa et al. } \\
\text { [6] }\end{array}$ & $\begin{array}{l}\text { Vivid images of pipes, tubing, toolmaking } \\
\text { machinery, and front view of a church }\end{array}$ & days & Neurologic insult during CABG & Spontaneous resolution \\
\hline 1991 & Fisher et al. & $\begin{array}{l}\text { Colortul, litelike images of Individual letters, } \\
\text { landscapes, and football players }\end{array}$ & $\begin{array}{l}\text { Eleven } \\
\text { days }\end{array}$ & Atropine toxicity & Spontaneous resolution \\
\hline 1991 & $\begin{array}{l}\text { Fisher et al. } \\
\text { [8] }\end{array}$ & $\begin{array}{l}\text { Colorful images of books, snowy landscapes, and } \\
\text { various animals }\end{array}$ & $\begin{array}{l}\text { Four } \\
\text { hours }\end{array}$ & Lidocaine use & Spontaneous resolution \\
\hline
\end{tabular}

\section{TABLE 1: Published case reports describing closed-eye visual hallucinations}

CABG: coronary artery bypass graft

Our patient experienced closed-eye visual hallucinations preceding severe alcohol withdrawal. While visual hallucinations are known during alcohol withdrawal, prodromal closed-eye visual hallucinations such as those described in our case have not been reported [14]. The patient also denied any prior occurrence of closed-eye visual hallucinations during polysubstance use or withdrawal. Finally, we considered that the closed-eye visual hallucinations might have occurred due to hepatic encephalopathy. However, the patient's ammonia levels were normal when he reported closed-eye visual hallucinations, despite moderate-to-severe transaminitis. His blood ammonia levels became abnormal the following day, coinciding with the onset of severe delirium attributed to acute hepatitis. Finally, given the patient's initial suicidality and eventual severe agitation, we considered whether the visual hallucinations might have occurred in the setting of psychosis. However, the patient's hallucinations were impersonal, unrelated to his thoughts or feelings, and did not speak to him directly. This contrasts with psychotic hallucinations, which are usually either egodystonic or ego-syntonic and interactive [15]. Furthermore, visual hallucinations attributed to psychosis are often accompanied by other features of psychosis such as delusions, disorganized thought processes, and behavior [15]. None of these were reported or observed when the patient was experiencing closed-eye visual hallucinations.

The closed-eye visual hallucinations reported by our patient are similar to the ones previously described in a few limited cases, distinct from the visual hallucinations described in Charles Bonnet syndrome or psychotic disorders [3-8]. Additional research is required to elucidate the mechanism, etiologies, and clinical implications of this curious phenomenon.

\section{Conclusions}

In contrast to the less complex and geometric visual hallucinations associated with visual impairment described in Charles Bonnet syndrome, or the interactive and disturbing visual hallucinations of psychosis, closed-eye visual hallucinations are vivid, animated yet impersonal, and are not always associated with delirium. As evidenced in this case study, closed-eye visual hallucinations may precede severe delirium. In the setting of alcohol withdrawal, the perceptual disturbance may resolve with treatment of underlying pathology. However, further research is necessary to elucidate the mechanism, specific etiology, and clinical implications of this intriguing phenomenon for prompt diagnosis and timely administration of life-saving treatment.

\section{Additional Information \\ Disclosures}

Human subjects: Consent was obtained or waived by all participants in this study. Conflicts of interest: In compliance with the ICMJE uniform disclosure form, all authors declare the following: Payment/services info: All authors have declared that no financial support was received from any organization for the submitted work. Financial relationships: All authors have declared that they have no financial 
relationships at present or within the previous three years with any organizations that might have an interest in the submitted work. Other relationships: All authors have declared that there are no other relationships or activities that could appear to have influenced the submitted work.

\section{Acknowledgements}

SD conceived the study, participated in data collection, drafted the manuscript, and managed the publication process. AT conceived the study, participated in data collection, edited the manuscript, and provided direct patient care. AS interpreted the data, proofread the final manuscript, provided direct patient care, and supervised the project. All authors read and approved the final manuscript. Additionally, we want to thank Dr. Stanley Safier and Dr. Adela Gerolemou for taking care of the patient and providing treatment supervision.

\section{References}

1. Teeple RC, Caplan JP, Stern TA: Visual hallucinations: differential diagnosis and treatment . Prim Care Companion J Clin Psychiatry. 2009, 11:26-32. 10.4088/pcc.08r00673

2. Schadlu AP, Schadlu R, Shepherd JB 3rd: Charles Bonnet syndrome: a review . Curr Opin Ophthalmol. 2009, 20:219-22. 10.1097/ICU.0b013e328329b643

3. Peck T, Mercogliano C, York E: Closed-eye visualizations in the setting of hyponatremia . Case Rep Med. 2018, 2018:5127917. 10.1155/2018/5127917

4. Weinschenk K, Schwartz AC: A case report of closed-eye visual hallucinations . Psychosomatics. 2011, 52:867. 10.1016/j.psym.2010.11.008

5. Otomo S, Sugita M, Yano T: Visual hallucinations on eye closure after orthopedic surgery under general anesthesia. J Anesth. 2008, 22:439-42. 10.1007/s00540-008-0665-8

6. Eissa A, Baker RA, Knight JL: Closed-eye visual hallucinations after coronary artery bypass grafting . J Cardiothorac Vasc Anesth. 2005, 19:217-9. 10.1053/j.jvca.2005.01.001

7. Fisher CM: Visual hallucinations on eye closure associated with atropine toxicity. A neurological analysis and comparison with other visual hallucinations. Can J Neurol Sci. 1991, 18:18-27. $10.1017 / \mathrm{s} 0317167100031255$

8. Fisher CM: Visual hallucinations and racing thoughts on eye closure after minor surgery . Arch Neurol. 1991, 48:1091-2. 10.1001/archneur.1991.00530220113031

9. Ohayon MM, Priest RG, Caulet M, Guilleminault C: Hypnagogic and hypnopompic hallucinations: pathological phenomena?. Br J Psychiatry. 1996, 169:459-67. 10.1192/bjp.169.4.459

10. Teunisse RJ, Cruysberg JR, Hoefnagels WH, Verbeek AL, Zitman FG: Visual hallucinations in psychologically normal people: Charles Bonnet's syndrome. Lancet. 1996, 347:794-7. 10.1016/s0140-6736(96)90869-7

11. Jan T, del Castillo J: Visual hallucinations: Charles Bonnet syndrome. West J Emerg Med. 2012, 13:544-7. 10.5811/westjem.2012.7.12891

12. Siegel RK: Hallucinations. Sci Am. 1977, 237:132-40. 10.1038/scientificamerican1077-132

13. Silvanto J, Cowey A, Lavie N, Walsh V: Striate cortex (V1) activity gates awareness of motion . Nat Neurosci. 2005, 8:143-4. 10.1038/nn1379

14. Kattimani S, Bharadwaj B: Clinical management of alcohol withdrawal: a systematic review . Ind Psychiatry J. 2013, 22:100-8. 10.4103/0972-6748.132914

15. Cummings JL, Miller BL: Visual hallucinations. Clinical occurrence and use in differential diagnosis . West J Med. 1987, 146:46-51. 